\title{
A Better Method for Evaluating Learning Performance Based on Information Entropy and Dynamic Accumulation Level
}

\author{
Li-Ling Yang \\ Economics \& Management College \\ Zhaoqing University \\ Zhaoqing, China
}

\author{
Chieh-Wen Hsu \\ Economics \& Management College \\ Zhaoqing University \\ Zhaoqing, China
}

\begin{abstract}
In general, most of the learning assessment measures students' learning effectiveness by arithmetic average score or weighted average score. Although the assessment process is simple and easy to understand, it is not flexible and tends to ignore the real situation in the learning process. Therefore, it is unable to effectively describe whether progress, regression or grade distribution is stable in the learning process. This study puts forward "dynamic accumulation level" for learning evaluation, calculates the dynamic accumulation of the examination results through exponential smoothing method, and introduces "information entropy" to measure students' learning stability and learning level. Moreover, this paper promotes the thesis of dynamic stability evaluation method, which analyzes more realistically and precisely on students' learning condition and performance. With the dynamic stability evaluation method, teachers may timely adjust their teaching skills and counsel students in a more effective way to enhance the quality of learning.
\end{abstract}

Keywords-information entropy; dynamic accumulation level; learning performance

\section{INTRODUCTION}

The assessment of academic performance is to judge and grade the students' learning performance and the level of reaching their learning goals. The purpose of teaching evaluation is not only evaluating students' learning achievements, but also providing references for teachers to modify and improve teaching methods. The traditional teaching evaluation method doesn't inform students' learning performance, such as progressive degree or stability degree. For example, in a semester, student A scores 100, 80 and 60; student B scores 60,80,100, and their semester scores are both 80 , which can't reflect the learning process: student B has improved and student $\mathrm{A}$ is moving backward. In the book Educational Psychology, Zhang and Lin proposed that for a long time, schools have adopted the approach of assessing students' learning performance by semester scores or even letting one exam take place, which is unfair and nonobjective. Accordingly, how to evaluate teaching quality scientifically is the key project to school teaching [1].

Zhang mentioned that learning process is a continuous and relevant process. Psychologists usually regard learning as the process rather than the result of behavior change [2]. Pike pointed out that teaching evaluation should not only provide learning performance, but also help proposing teaching plans for the next stage based on the understanding of learning process [3]. White considered it is extremely complicated and difficult to evaluate teaching quality correctly [4]. Hua propose a more thorough and fair teaching evaluation, he compared process assessment and summative assessment on examination of college students' physical education learning effect applied by experimental method, investigation method, mathematical statistics or other research methods, identifying that the procedural evaluation is more objective and rational than summative evaluation, with a more outstanding feedback effect [5]. Zheng et al. also proposed that dynamic assessment can understand students' learning status better [6].

In terms of the theoretical calculation of information entropy, the literature adopts the concept of information entropy proposed by Shannon has been widely applied in the field of social economy [7]. In the practical application process, Liu et al., proposed a basic method of information entropy calculation, indicating that information entropy and standard deviation can reflect the degree of data confusion, but there is a significant difference between the two [8]. Luo calculated the weight of assessment indexes with the information entropy theory, showing that the weight obtained by information entropy can fully reflect the difference between data, which was proved in several cases [9]. Jin et al., applied entropy weight method to evaluate the performance of higher vocational education. The size of entropy weight can reflect the roles of different indexes in the evaluation [10]. Zheng et al., also applied the information entropy theory to measure the change of students' learning "stability level" in learning process [6].

This study will further revise the dynamic evaluation model proposed by Zheng et al. [6] Taking a teaching and learning assessment in college statistics class as an example. Evaluating students' learning "dynamic accumulation level" and "stability level" to understand the individual learning process and stability helps improve the teaching and learning quality. 


\section{LEARNING PERFORMANCE EVALUATION METHOD}

\section{A. Dynamic Accumulation Level}

Suppose $\mathrm{n}$ tests are held in the teaching process, and the sequence of tests results is $\left\{y_{1}, y_{2}, \ldots, y_{n}\right\}$, and its dynamic accumulation level is defined as:

$\mathrm{S}=\alpha^{n} y_{1}+\alpha^{n-1} y_{2}+\cdots+\alpha y_{n}, \quad$ including $0<\alpha<1$, said at a different time of test results to give different weights, In $\mathrm{n}$ tests, the smallest weight on the first test, the later the bigger the weight, show the score on the last test is better than the score on the previous one, the greater the dynamic cumulative effectiveness. It means that dynamic accumulation level emphasizes the energy of continuous progress in learning. In the above equation, the test scores can be calculated by using exponential smoothing method to calculate the weight value of each test scores, assuming that the dynamic accumulation level of the $t$ test is $S_{t}$ :

$$
\begin{aligned}
S_{t} & =\alpha y_{t}+(1-\alpha) S_{t-1} \\
& =a y_{t}+(1-\alpha)\left[a y_{t-1}+(1-\alpha) S_{t-1}\right] \\
& =a y_{t}+a(1-\alpha) y_{t-1}+a(1-\alpha)^{2} y_{t-2}+\cdots+a(1-\alpha)^{t-2} y_{2}+(1-a)^{t-1} y_{1}
\end{aligned}
$$

From the above equation, it can be known that $S_{t}$ is a linear combination of test scores $y_{t}$, and the dynamic cumulative level weight is calculated by multiplying each test scores by different weights respectively. The weight of the first test scores is the minimum, and the geometric series increases in order. The last test scores have the maximum weight, which are

$$
(1-\alpha)^{t-1}, \alpha(1-\alpha)^{t-2}{ }_{,}, \alpha, \alpha(1-\alpha)^{2}, \alpha(1-\alpha), \alpha
$$

The sum of the weights is

$$
\begin{aligned}
& (1-\alpha)^{t-1}+\alpha(1-\alpha)^{t-2}+\cdots+\alpha(1-\alpha)^{2}+\alpha(1-\alpha)+\alpha \\
& =(1-\alpha)^{t-1}+\left[1-(1-\alpha)^{t-1}\right]=1
\end{aligned}
$$

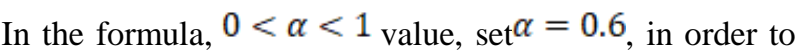
make a more significant difference between the test results before and after.

\section{B. Stability Level}

1) The information entropy: "Entropy" is a physical phenomenon in thermodynamics indicating the uniformity of energy distribution or the disorder of particles in a system. Shannon (1948) introduced the concept of entropy into the category of information theory, gave a new meaning to entropy and named it "information entropy". It measures the concept of uncertainty of system information source and defines it as the probability of occurrence of discrete random events. The higher the information entropy, the more chaotic a group of information, information entropy can be said to be a measure of the degree of ordering.

Definition of information entropy: suppose that $\mathrm{X}$ is a discrete random variable, $\mathrm{X}=\left\{x_{1}, x_{2}, \ldots, x_{n}\right\}$

$$
\text { Set } p_{i}=\mathrm{P}\left\{\mathrm{X}=x_{i}\right\}, \quad \forall \mathrm{x} \in \mathrm{X}, \mathrm{I}(\mathrm{x})=-\log \mathrm{P}\{\mathrm{X}=\mathrm{x}\}=-\log \left(p_{i}\right) \text {, }
$$

At this time $\mathrm{I}(\mathrm{x})_{\text {can }}$ be considered as provided by the amount of information $\mathrm{x}$. Information is used to describe the amount of information needed to eliminate the uncertainty at the random variable $X=x$, when the probability of $p_{i}=\mathrm{P}\left\{\mathrm{X}=x_{i}\right\}$ is high, it means that the probability of this event is high, the uncertainty factor is small, the uncertainty can be eliminated without much information, so the information of $I(x)$ is small. Information entropy $H(X)$ is the mathematical expectation of information $-\log P\{X=x\}$, that is, the sum of information at all $\mathrm{X}=\mathrm{x}$ points. Therefore, information entropy is used to describe the size of the total information needed to eliminate the uncertainty of random variable $X$. Therefore, it is expressed by the expectation of information quantity $\mathrm{I}(\mathrm{x})$, that is, the information entropy is defined as follows:

$$
\mathrm{H}(\mathrm{X})=\mathrm{E}(\mathrm{I}(\mathrm{X}))=-\mathrm{c} \sum_{i=1}^{n} p_{i} \log \left(p_{i}\right)
$$

Where, $c$ is the proportionality coefficient $\forall p_{i}=0$ or $1, p_{i} \log \left(p_{i}\right) \equiv 0, i=1,2, \ldots, n$. If the probability is 1 or 0 , it means that there is little information contained in the deterministic event, that is, information entropy $\mathrm{H}(\mathrm{X})=0$, which means there is no uncertain information. When $p=0.5$, the uncertain information entropy is the largest. The minus sign here is just to keep entropy positive or zero.

$$
l=\frac{100}{m},
$$
divisions, the length of each interval is 1 , and the Kth subinterval of $\mathrm{m}$ intervals is marked as $p_{k}$, indicating the frequency of record results appearing in the Kth interval $\left[\frac{100(k-1)}{m}, \frac{100 k}{m}\right]$.

Information entropy is defined as the fluctuation level:

$$
\Lambda(\mathrm{p})=-\frac{1}{\log (m)} \sum_{k=1}^{m} p_{k} \log \left(p_{k}\right)
$$

Among them, the $\sum_{k=1}^{m} p_{k}=1,0 \leq \Lambda(\mathrm{p}) \leq 1$, by $\mathrm{G}=1-\Lambda(\mathrm{p})$ measure stable level degree It can be seen from the definition of entropy that the greater the variation degree of information source, the greater the entropy, the greater the uncertainty information provided by this indicator, and the smaller the stability level. Conversely, the less variable the source, the greater the level of stability will be.

2) Adjusted stability level: Stability level just shows the score in a certain area of stability, but not emphasize the stability degree is in high partition (m) or low partition (1), the purpose of adjusting wants to maintain stability in the high partition progress than in the low steady progress can be the difference, and there will be a good learning performance, so the stability level of the partition should be greater than in the stable level of low area, the original $m$ range, should be given different weights, hope in a stability level, Maximum range to give maximum weight of $m / m$, the second highest 
time interval given the second largest weight of $(m-1) / m$, so on to a minimum range, to give the minimum weight of $1 / m$, but in the process of adjusting level fluctuations which give weight and stability level just weights on the contrary, the highest range to give maximum weight of $1 / \mathrm{m}$, the second highest time interval given the large weight of $2 / \mathrm{m}$, so on to a minimum range, to give the minimum weight of $m / m$

Therefore, each interval was given different weights $\underline{(m-k-1)}$

$m$, and the adjusted fluctuation level was

$$
\begin{aligned}
& \operatorname{MZ}(m)=-\frac{1}{\log (m)} \sum_{k=1}^{m} p_{k} \log \left(p_{k}\right) \times \frac{(m-k-1)}{m} \\
& \operatorname{MG}(\mathrm{m})=\left\{\begin{array}{c}
1-M Z(m), 0 \leq p_{k}<1, k=1,2, \ldots, m . \\
\frac{k}{m}, \quad p_{k}=1, k=1,2, \ldots, m .
\end{array}\right.
\end{aligned}
$$
measuring points at the same time stability and placement of interval position degree level.

3) Dynamic learning stability level: Setting $\Psi$ (n) for $n$ score on the test and dynamic learning stability level, $\Psi$ (n) = $\mathrm{S} * \mathrm{MG}$. where, $\mathrm{S}$ and $\mathrm{MG}$ respectively $\mathrm{n}$ dynamic accumulation level and adjusted stability level, student learning condition depends on the dynamic accumulation level and adjusted stability level of the product. If the dynamic accumulation level in the learning process is high, and the adjusted stability level is also high, the product of two factors gets a high dynamic learning stability level. It means that students' performance continues to improve during the learning process and the results are distributed in high grades. Dynamic learning stability level can be used to measure the excellent learning performance of the students.

\section{RESULTS AND DISCUSSION}

This study takes 50 accounting students' statistics learning evaluation in a business school as the object of the research and evaluates them dynamically with a method mentioned above in this study. Learning performance evaluation is as follows:

\section{A. Dynamic Accumulation Level and Stability Level Calculation}

The dynamic accumulation level is calculated based on $\alpha=0.6$, according to equation (1) which is the arithmetic mean of five statistics test scores (Test 1 , Test 2, Test 3, Test 4, Test 5). While the stability level (MG) and adjusted fluctuation level (MZ) are calculated by formula (4) with the method of dividing the scores into five groups, each with 20 points. At last, the dynamic learning stability level is calculated by dynamic accumulation level times adjusted stability level. ("Table I")

\begin{tabular}{|c|c|c|c|c|c|c|c|c|c|c|}
\hline No & $\begin{array}{c}\text { Test } \\
1\end{array}$ & $\begin{array}{c}\text { Test } \\
2\end{array}$ & $\begin{array}{c}\text { Test } \\
\mathbf{3}\end{array}$ & $\begin{array}{c}\text { Test } \\
4\end{array}$ & $\begin{array}{c}\text { Test } \\
5\end{array}$ & AVE & $\begin{array}{c}\text { DAL } \\
\text { (1) }\end{array}$ & SL & Adj-SL(2) & $\begin{array}{c}\text { DLSL } \\
=(\mathbf{1}) *(2)\end{array}$ \\
\hline 1 & 81 & 88 & 90 & 85 & 88 & 86.40 & 87.29 & 1.00 & 1.00 & 87.29 \\
\hline 2 & 79 & 84 & 88 & 88 & 89 & \begin{tabular}{|l|}
85.60 \\
\end{tabular} & 88.22 & \begin{tabular}{|l|}
0.69 \\
\end{tabular} & \begin{tabular}{|l|}
0.90 \\
\end{tabular} & 79.20 \\
\hline 3 & 70 & 88 & 95 & 90 & 93 & 87.20 & 91.69 & 0.69 & 0.90 & 82.32 \\
\hline 4 & 53 & 88 & 78 & 75 & 70 & 72.80 & 72.22 & 0.41 & 0.76 & 55.17 \\
\hline 5 & 75 & \begin{tabular}{|l|}
69 \\
\end{tabular} & 76 & 86 & 89 & \begin{tabular}{|l|}
79.00 \\
\end{tabular} & 85.91 & 0.58 & \begin{tabular}{|l|}
0.88 \\
\end{tabular} & 75.45 \\
\hline 6 & 63 & 76 & 83 & 86 & 89 & \begin{tabular}{|l|}
79.40 \\
\end{tabular} & 86.54 & 0.58 & 0.87 & 75.36 \\
\hline 7 & 54 & 76 & 90 & 88 & 91 & \begin{tabular}{|l|}
79.80 \\
\end{tabular} & 88.66 & 0.41 & 0.76 & 67.55 \\
\hline 8 & 66 & 82 & 85 & 88 & 91 & 82.40 & 88.72 & 0.69 & 0.90 & 79.65 \\
\hline 9 & 68 & 84 & 78 & 86 & 89 & 81.00 & 86.49 & 0.58 & 0.87 & 75.32 \\
\hline 10 & 18 & 70 & 85 & 86 & 89 & 69.60 & 85.35 & 0.41 & 0.68 & 58.20 \\
\hline 11 & 80 & 91 & 90 & 93 & 98 & 90.40 & 95.30 & 0.69 & 0.90 & 85.56 \\
\hline 12 & 51 & 42 & 0 & 82 & 80 & \begin{tabular}{|l|}
51.00 \\
\end{tabular} & 70.60 & 0.17 & 0.54 & 38.36 \\
\hline 13 & 58 & 80 & 90 & 88 & 91 & 81.40 & 88.92 & 0.41 & 0.76 & 67.75 \\
\hline \begin{tabular}{|l|}
14 \\
\end{tabular} & 43 & 57 & 64 & 84 & 0 & \begin{tabular}{|l|}
49.60 \\
\end{tabular} & 29.59 & 0.17 & 0.54 & 16.08 \\
\hline 15 & 41 & 78 & 78 & 86 & 89 & \begin{tabular}{|l|}
74.40 \\
\end{tabular} & 85.57 & 0.34 & 0.74 & 63.61 \\
\hline 16 & 78 & 66 & 83 & 88 & 91 & 81.20 & 88.22 & 0.58 & 0.87 & 76.82 \\
\hline 17 & 65 & 70 & 80 & 86 & 89 & 78.00 & 86.07 & 0.58 & 0.88 & 75.60 \\
\hline 18 & 82 & 88 & 95 & 90 & 93 & 89.60 & 92.00 & 1.00 & 1.00 & 92.00 \\
\hline 19 & 63 & 51 & 78 & 86 & 89 & 73.40 & 85.10 & 0.34 & 0.74 & 63.26 \\
\hline 20 & 25 & 44 & 31 & 40 & 35 & 35.00 & 35.91 & 0.69 & 0.79 & 28.41 \\
\hline \begin{tabular}{|l|}
21 \\
\end{tabular} & 60 & 62 & 55 & 82 & 85 & \begin{tabular}{|l|}
68.80 \\
\end{tabular} & \begin{tabular}{|l|}
79.88 \\
\end{tabular} & 0.34 & \begin{tabular}{|l|l|}
0.74 \\
\end{tabular} & \begin{tabular}{|l|l|}
58.93 \\
\end{tabular} \\
\hline 22 & 66 & 82 & 65 & 84 & 87 & 76.80 & 83.44 & 0.58 & 0.87 & 72.66 \\
\hline 23 & 15 & 39 & 60 & 75 & 85 & 54.80 & 76.64 & 0.00 & 0.40 & 30.66 \\
\hline \begin{tabular}{|l|}
24 \\
\end{tabular} & 80 & 80 & 93 & 90 & 93 & \begin{tabular}{|l|}
87.20 \\
\end{tabular} & 91.45 & 0.58 & \begin{tabular}{|l|l|}
0.87 \\
\end{tabular} & 79.63 \\
\hline \begin{tabular}{|l|}
25 \\
\end{tabular} & 28 & 30 & 55 & 45 & 53 & 42.20 & 49.75 & 0.58 & 0.70 & 35.00 \\
\hline 26 & 75 & 69 & 93 & 90 & 93 & 84.00 & 90.90 & 0.58 & 0.87 & 79.16 \\
\hline \begin{tabular}{|l|}
27 \\
\end{tabular} & 53 & 48 & 83 & 88 & 91 & \begin{tabular}{|l|}
72.60 \\
\end{tabular} & 86.89 & 0.58 & 0.83 & 71.71 \\
\hline 28 & 59 & 65 & 78 & 86 & 89 & 75.40 & 85.53 & 0.34 & 0.74 & 63.58 \\
\hline \begin{tabular}{|l|}
29 \\
\end{tabular} & 55 & 74 & 88 & 88 & 91 & \begin{tabular}{|l|}
79.20 \\
\end{tabular} & 88.42 & 0.41 & 0.76 & 67.37 \\
\hline 30 & 45 & 67 & 73 & 86 & 89 & 72.00 & 84.77 & 0.34 & 0.74 & 63.02 \\
\hline 31 & 65 & 69 & 75 & 86 & 89 & 76.80 & 85.55 & 0.58 & 0.88 & 75.14 \\
\hline 32 & 61 & 60 & 72 & 86 & 55 & 66.80 & 64.42 & 0.34 & 0.73 & 47.17 \\
\hline 33 & 75 & 88 & 93 & 90 & 93 & 87.80 & 91.63 & 0.69 & 0.90 & 82.26 \\
\hline 34 & 34 & 41 & 63 & 84 & 87 & \begin{tabular}{|l|}
61.80 \\
\end{tabular} & 80.85 & 0.17 & 0.59 & 48.06 \\
\hline 35 & 45 & 63 & 85 & 88 & 91 & 74.40 & 87.45 & 0.41 & 0.76 & 66.63 \\
\hline 36 & 80 & 85 & 80 & 85 & 80 & 82.00 & 81.39 & 0.58 & 0.88 & 71.48 \\
\hline 37 & 80 & 70 & 75 & 84 & 87 & \begin{tabular}{|l|l|}
79.20 \\
\end{tabular} & 84.30 & 0.58 & 0.88 & 74.04 \\
\hline 38 & 80 & 91 & 93 & 90 & 93 & 89.40 & 91.87 & 0.69 & 0.90 & 82.48 \\
\hline 39 & 17 & 20 & 15 & 15 & 18 & 17.00 & 17.04 & 1.00 & 0.20 & 3.41 \\
\hline 40 & 45 & 67 & 73 & 86 & 89 & 72.00 & 84.77 & 0.34 & 0.74 & 63.02 \\
\hline 41 & 43 & 44 & 78 & 86 & 89 & 68.00 & 84.32 & 0.34 & 0.74 & 62.21 \\
\hline 42 & 70 & 74 & 71 & 86 & 89 & 78.00 & 85.49 & 0.58 & 0.88 & 75.08 \\
\hline \begin{tabular}{|l|}
43 \\
\end{tabular} & 81 & 81 & 93 & 90 & 93 & 87.60 & 91.51 & 1.00 & 1.00 & 91.51 \\
\hline \begin{tabular}{|l|}
44 \\
\end{tabular} & 53 & 48 & 88 & 88 & 91 & 73.60 & 87.37 & 0.58 & 0.83 & 72.10 \\
\hline \begin{tabular}{|l|}
45 \\
\end{tabular} & 91 & 77 & 62 & 43 & 30 & 60.60 & 39.56 & 0.17 & 0.59 & 23.30 \\
\hline \begin{tabular}{|l|}
46 \\
\end{tabular} & 45 & 19 & 63 & 51 & 65 & 48.60 & \begin{tabular}{|l|}
59.17 \\
\end{tabular} & 0.34 & 0.57 & 33.86 \\
\hline \begin{tabular}{|l|}
47 \\
\end{tabular} & 28 & 30 & 67 & 58 & 70 & 50.60 & 64.22 & 0.34 & 0.61 & 38.96 \\
\hline \begin{tabular}{|l|}
48 \\
\end{tabular} & 55 & 40 & 67 & 44 & 48 & 50.80 & 48.74 & 0.41 & 0.65 & 31.47 \\
\hline \begin{tabular}{|l|}
49 \\
\end{tabular} & 67 & 62 & 50 & 39 & 35 & \begin{tabular}{|l|l|}
50.60 \\
\end{tabular} & 39.26 & 0.34 & 0.61 & 23.82 \\
\hline \begin{tabular}{|l|}
50 \\
\end{tabular} & 50 & 89 & 68 & 74 & 78 & 71.80 & 75.79 & $\mid 0.41$ & 0.76 & 57.89 \\
\hline
\end{tabular}

TABLE I. Learning Performance Evaluation and Grading

${ }^{\text {a. }}$ Note: AVE: Average scores; DAL: Dynamic accumulation level; SL: Stability level Adj_SL: AdjStability level; DLSL: Dynamic learning stability level 


\section{B. Grading of Learning Performance}

The study divides students' learning performance into three groups with the method of K-means cluster analysis. As is shown in "Fig.1", Group I, graded as 'Excellent', the dynamic accumulation level is roughly between 75 and 100 Group II, graded as 'Fine', the level is between 50 and 75 , while Group III, graded as 'Falling behind', the level is below 50

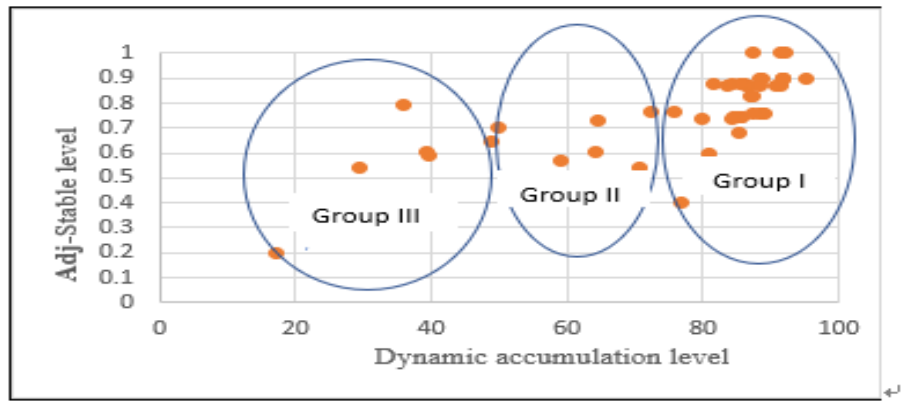

Fig. 1. Dynamic accumulation and adjustment of stability level distribution

\section{Group I (Excellent)}

There were 38 students in Group I, graded as excellent students. The average of dynamic accumulation level was 86.59 , and the average of dynamic stability level was 0.82 .

\section{Group II (Fine)}

There were 7 students in Group II, graded as fine students. The average of dynamic accumulation level was 61.30 , and the average of dynamic stability level was 0.65 .

\section{Group III (Falling behind)}

There were 5 students in Group III, graded as falling behind students. The average of dynamic accumulation level was 32.27 , and the average of dynamic stability level was 0.71 .

\section{Discussion}

1) Analysis effectiveness of dynamic accumulation level: The level of dynamic accumulation emphasizes on the progress of learning as it is a continuous process. The learning condition affects the learning performance; the more you make progress and keep grades, the higher the dynamic accumulation level will be. Based on the dynamic cumulative level shown in "Table I", there's an example:

By looking at student No. 47 and No.49 test scores, their average scores of five tests are both 50.6 points, but the dynamic accumulation level are different, respectively being 64.22 and 39.26. With the view of average scores, two students' learning performance is the same. However, in fact, under evaluation with dynamic accumulation level, we found that student No.47 keeps making progress on learning while student No.49 is falling behind. Therefore, dynamic accumulation level shows more practically and rationally that student No.47 has better performance than student No.49.

With the traditional average score method, student No. 47 and No. 49 has the same learning performance; while through the dynamic accumulation level evaluation, and student No. 47 kept improving and had better performance than student No. 49.

2) Analysis effectiveness of stability level: The study not only focuses on the importance of the cumulative effect in the learning process, but also the stability of learning. We expect student to grow stability, not to grow in waves. Therefore, in addition to learning progress, but also to have a stable degree of learning, in order to achieve a good real learning level. In order to motivate the student with high scores stability, the interval with high scores should have larger stable weight. The following examples are given to illustrate the effectiveness of stability level evaluation:

The dynamic accumulation level of student No.1 and student No.7 is 87.29 and 88.66 respectively, and the stability level is 1.0 and 0.76 respectively. Without considering the stability level, student No.7 achieved better learning progress.

However, when stability effectiveness was added, the real learning level of the two was 87.29 and 67.55, respectively. Because student No.1 has a high level of stable learning makes his learning level higher than student No.7.

As shown in table 1, student No.1 scored five times, four times in the interval of the fourth grade and one time in the interval of the fifth grade. Student No.7 scored five times, including once in the interval of level 3 , twice in the interval of level 4, and twice in the interval of level 5. If the weights of all intervals are the same, student No.1 has a higher stability level of 1.0 due to a four-fifths high frequency in the fourth level range. Although student No.7 score falls in the range of high score, his score is scattered in three intervals, which makes his stability level lower 0.76 .

If only the dynamic accumulation level is considered, student No.7 is better than student No.1; when we add stability level, the higher the weight of stability, student No.1 is better than student No.7.

3) Analysis effectiveness of adjusted stability level: Student No.18 and student No. 39 have a stability level of 1 , 
0 respectively, and their scores both are high stability. In fact, student No.18 is stable on the high partition; versus student No.39 is stable on the low partition. It is impossible to distinguish the difference between high and low zone stability for the real learning performance. Therefore, when adding the computation adjusted stability level of 1.0 and 0.2 respectively, should be shown that the grade of student No.18 is highly stable and falls in the high division.

4) Analysis effectiveness of dynamic learning stability level: The average score of student No.1 and student No.2 was 87.29 and 88.22 respectively. The dynamic learning stability level was 87.29 and 79.20 respectively. Although student No.2 is better on average, if based on the dynamic stable learning level, student No.1 has a better learning performance than No.2. In fact, student No.1 scores above 80 in each examination, his performance is getting better and more stable, while student No.2 sometimes scores below 80 , although the average score of student No.2 is higher, however, the overall performance of student No.1 is better.

Dynamic learning stability level is the product of the dynamic accumulation level and the adjusted stability level, so students' learning stability and learning level are considered at the same time. "Dynamic learning stability level" evaluation mode can more objectively and fairly evaluate student' learning performance and stability.

\section{CONCLUSION AND MANAGEMENT IMPLICATIONS}

\section{A. Conclusion}

This study demonstrates the value of "dynamic learning stability level method" and "arithmetic mean method" is different in evaluating the learning effect, showing that the process evaluation is more effective and reasonable than the summative evaluation. In present learning evaluation method, neither the arithmetic average nor the weighted average can highlight the influence of the progress and stability of the learning process. The whole learning process is a continuous process; learning should be continuous steady progress to have good results. Process assessment provides timely learning feedback, so teachers may be able to tutor students who are having difficulties in learning or encourage students who have already had good grades to keep working hard, which reinforces learning motivation. All in all, this dynamic teaching evaluation method can effectively improve teaching quality.

\section{B. Management Implications}

1) Establishing an effective teaching evaluation system: The "dynamic accumulation method" emphasizes the importance of continuous progress in the learning process. Through the dynamic accumulation level in the learning stage, the students' actual learning situation can be seen. If the student continues to progress, the dynamic accumulation level will keep increasing. Otherwise, the dynamic accumulation level will decrease. Second, the "adjusted stability level method" accentuates the importance of learning stability. If the academic performance is maintained in the high score zone, the higher the stability level will be. Otherwise, the adjusted stability level will be lower. To sum up, the "dynamic learning stability level" is combined with the dynamic accumulation and adjusted stability level, which means the higher the dynamic learning stability level, the students continue to make progress in study and maintain in high grades. It can improve the facts that summative evaluation only focuses on the result rather than the process of learning.

2) Improving teaching quality: "Dynamic learning stability level method" describes the students' learning process more accurately on either progression or regression and their learning stability. With the method, teachers can focus more on students who have been getting worse or fluctuating grades by giving them timely care and counselling. Through the method, students are encouraged to make continuously steady progress, while teachers to improve their teaching skills and quality with a better teacher-student interaction eventually.

\section{REFERENCES}

[1] C. S. Zhang, Q. S. Lin, Educational psychology, Taipei, 2000, pp. 373-382.

[2] C. S. Zhang, Psychology, Taipei Donghua, 2000, pp. 112-114.

[3] C. K. Pike, "A validation of an instrument designed to measure teaching effectiveness", Journal of Social Work Education, 34, 1998, pp. 261-271.

[4] E. M. White, "How to improve evaluation of teaching", Chronicle of Higher Education, 47, 2000, pp.11-21.

[5] Y. Hua, "Discussion on process evaluation of college physical education learning", Journal of Wuhan University of Physical Education, 39:4, 2005, pp. 111-112.

[6] D. Z. Zheng , F. R. Ou, Z.X. Lin, "Dynamic evaluation of learning effect and definition of students with poor learning quality", Journal of China University of Science and Technology, 45, 2010, pp. 127 142 .

[7] C. E. Shannon, "A Mathematical Theory of Communication". The Bell System Technical Journal, 27, 1948, pp.379-423.

[8] S. L. Liu, S. L. Zhu, E. U. Wang, "Calculation of information entropy of middle school physics grades", Journal of Lingnan Normal University, 38:3, 2017, pp. 73-78

[9] J. Luo, "Principles and examples of using information entropy to calculate the weight of evaluation indexes", Journal of Wuhan Textile University, 27:6, 2014, pp. 86-89.

[10] R. X. Jin, Mao Qiongzhi, S. Zhang, "Performance evaluation of higher vocational education in china based on ahp and entropy weight method", Journal of Finance and Accounting, 36, 2017, pp.59-66 